\title{
On the violation of the holographic viscosity versus entropy KSS bound in non-relativistic systems
}

\author{
A. Dobado, F.J. Llanes-Estrada ${ }^{a}$ \\ Departamento de Física Teórica I, Universidad Complutense de Madrid, 28040 Madrid, Spain \\ Received: 14 March 2007 / Revised version: 27 April 2007 / \\ Published online: 21 June 2007 - (C) Springer-Verlag / Società Italiana di Fisica 2007
}

\begin{abstract}
A computation of the quotient of shear viscosity and entropy density, or the Kovtun, Son and Starinets (KSS) number $\eta / s$, is performed in the non-relativistic and classical regime, first in chiral perturbation theory, and then in the $\mathrm{SO}(g+1) / \mathrm{SO}(g)$ non-linear sigma model in the large $g$ limit. Both are field theories stemming from a renormalizable sigma model, but, in spite of that, we explicitly calculate how one avoids the KSS bound by increasing the number of degenerate pions sufficiently. However, we argue that particle production could still keep the validity of the KSS bound in the weak sense. We also show how a large number of molecular isomers (which we estimate in terms of simple molecular properties) could avoid the bound in the strong sense. This might be possible with carbon based molecules. We finally argue that a measurement of $\eta / s$ in heavy-ion collisions might be turned into an upper bound on the number of hadron resonances.
\end{abstract}

PACS. 11.15.Pg; 12.38.Mh; 25.75.q; 51.20.+d

\section{Introduction}

There is considerable interest in devising or reporting on fluids with the lowest possible value of the shear viscosity to entropy density ratio, $\eta / s$. This ratio, dimensionless in natural units, and taken at zero chemical potential, is responsible for the damping of shear waves in a fluid with the dispersion relation $\omega+\mathrm{i} k^{2}(\eta / s T)=0$. A few years ago, Kovtun, Son and Starinets (KSS) [1] observed that in field theories that have a gravity dual in higher dimension through the holographic principle, the ratio could be estimated as a function of the metric coefficients near a "black brane":

$$
\frac{\eta}{s}=T f\left[g_{\alpha \beta}\right]
$$

and to their surprise, several feasible calculations with simple metrics $g_{\alpha \beta}$ consistently yielded the value $\eta / s=$ $1 /(4 \pi)$. The field theories dual to these gravity configurations are strongly coupled supersymmetric Yang-Mills theories, far removed from our current physical picture of the world. However, going to common substances whose viscosity and entropy density values are tabulated, one finds (see Fig. 1) that this ratio is at least an order of magnitude larger than $1 /(4 \pi)$. In the spirit of other dimensionless numbers characterizing fluid mechanics, such as the Reynolds or the Prandtl number, we can define (now in ar-

\footnotetext{
a e-mail: fllanes@fis.ucm.es
}

bitrary units) the KSS number as

$$
\mathrm{KSS}=\frac{k_{\mathrm{B}} \eta}{\hbar s} .
$$

Given that no fluid is known to have $\mathrm{KSS}<1 /(4 \pi)$, the authors conjectured that this value is a universal bound for the ratio (strong bound). Since then, several works have reported on values for the ratio in the range $0.08-$

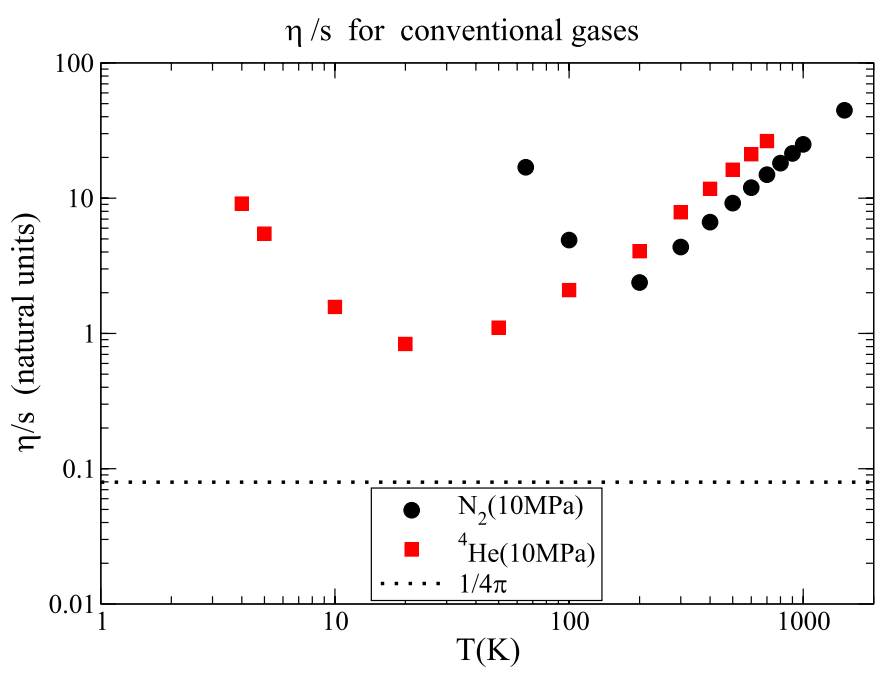

Fig. 1. $\eta / s$ for molecular nitrogen and helium gases together with the bound of Kovtun, Son and Starinets 
0.3 for the quark and gluon liquid produced at RHIC [3], 0.3 for cold, trapped atoms near a Feshbach resonance [4], and theoretical work has shown that the pion gas in the aftermath of a heavy-ion collision should respect the bound [5] (correcting earlier results from [6]), as also should the nuclear matter formed in possible strange stars [7].

As was stressed in [2], the KSS bound does not involve the speed of light $c$ and hence is non-trivial when applied to non-relativistic systems. It is not clear whether the bound is or is not appropriate for all such systems. The reason is that one can consider a gas with an increasing number of different species of molecules, so that the entropy density can be made arbitrary large, thus evading the bound. However, it can be argued this cannot happen in any respectable non-relativistic system coming from a genuine relativistic quantum field theory with a well defined ultraviolet (UV) completion (weak sense bound). In this work we will show how the bound can be violated by the non-relativistic classic limit of a $\mathrm{SO}(g+1) / \mathrm{SO}(g)$ non-linear sigma model (NLSM) for a sufficient large $g$. As is well known, this Lagrangian density is the effective field theory of the corresponding linear sigma model (LSM); that is, a renormalizable field theory (although the loophole of UV completion remains open, since the beta function is positive, one can call into question the relevance of this issue in the nonrelativistic limit. Further discussion can be found in the recent paper [8]).

In order to expose the violation, we will first consider the case of a non-relativistic classical pion gas described by chiral perturbation theory $(\chi \mathrm{PT})$ and then we will extend the result to the $\mathrm{SO}(g+1) / \mathrm{SO}(g)$ non-linear sigma model to see how the weak version of the bound can be avoided. Next we will comment on a possible way out for the KSS conjecture and finally we examine what one would need to achieve in molecular physics a system realized in nature violating the bound. Throughout the paper we distinguish the "KSS bound" (meaning $\eta / s>1 /(4 \pi)$ for an arbitrary system) from the "KSS conjecture" (the actual statement that this bound could strictly hold for relativistic field theories in some more or less strong form depending on additional assumptions).

\section{The bound applies for a non-relativistic pion system}

As is well known, the low-energy dynamics of pions can be described by $\chi \mathrm{PT}[9,10]$. At the lowest order this amounts to the NLSM based on the symmetry group $\mathrm{SU}(2)_{\mathrm{L}} \times$ $\mathrm{SU}(2)_{\mathrm{R}}$ being spontaneously broken to $\mathrm{SU}(2)_{\mathrm{L}+\mathrm{R}}$. As a consequence the fields have as domain the coset manifold $\mathrm{SU}(2)_{\mathrm{L}} \times \mathrm{SU}(2)_{\mathrm{R}} / \mathrm{SU}(2)_{\mathrm{L}+\mathrm{R}}=\mathrm{SO}(4) / \mathrm{SO}(3)=S^{3}$. This symmetry scheme is manifest in the Lagrangian for the pion fields:

$$
\mathcal{L}_{\chi}=\frac{1}{2} g_{a b} \partial_{\mu} \pi^{a} \partial^{\mu} \pi^{b}+m^{2} f^{2} \sqrt{1-\pi^{2} / f^{2}},
$$

where the coset metric is given by

$$
g_{a b}=\delta_{a b}+\frac{\pi_{a} \pi_{b}}{f^{2}-\pi^{2}},
$$

with $f \simeq 92 \mathrm{MeV}, m \simeq 138 \mathrm{MeV}, a, b$ running from $a, b=1$ to $a, b=g=3$ and $\pi^{2}=\pi^{a} \pi^{a}$. Note that we have added an explicit symmetry breaking term proportional to $m^{2}$ to the NLSM Lagrangian in order to take into account the pion mass $m$.

From the above Lagrangian it is possible to compute the elastic scattering amplitude of the pion, which can be written as $T_{a b c d}=A(s, t, u) \delta_{a b} \delta_{c d}+\ldots$, where we have not explicitly shown the crossing terms. At low energies, which is the relevant limit for the non-relativistic regime, the amplitude is given by the Weinberg low energy theorem: $A=\left(s-m^{2}\right) / f^{2}{ }^{1}$ The corresponding averaged cross section in the non-relativistic limit is

$$
\sigma=\frac{23 m^{2}}{384 \pi f^{4}}=\pi R^{2} .
$$

As this cross section is energy independent in this nonrelativistic limit we have introduced the effective pion radius $R$.

On the other hand the well-known viscosity of a classical, hard-sphere gas grows moderately for low temperature as a square root,

$$
\eta=\frac{5 \sqrt{m T}}{16 \sqrt{\pi} R^{2}}
$$

in terms of the hard-sphere radius $R$ and mass $m$. Thus we can use the above results to compute the viscosity of a nonrelativistic classical gas of pions, which is given by

$$
\eta_{\chi}=\frac{120 \pi^{3 / 2} f^{4}}{23 m^{3 / 2}} \sqrt{T} .
$$

The entropy density of a Bose gas with $g$ different components is conveniently taken as

$$
s=\frac{g}{6 \pi^{2} T^{2}} \int_{9}^{\infty} p^{4} \mathrm{~d} p \frac{E-\mu}{E} \frac{\mathrm{e}^{\beta(E-\mu)}}{\left[\mathrm{e}^{\beta(E-\mu)}-1\right]^{2}} .
$$

Note that we have introduced a chemical potential $\mu \leq m$. Strictly speaking this requires that the number of bosons (pions in our case) is conserved by the interactions. However, from the $\chi \mathrm{PT}$ Lagrangian we clearly see that interactions with any even number of pions are allowed. In particular 2 to 4 pion reactions are present whenever the center of mass energy is larger than the four pion threshold located at $s=16 \mathrm{~m}^{2}$. In spite of that, in the low energy (nonrelativistic) regime we are considering here, these non pion number conserving processes are completely suppressed, and thus it is possible to introduce an effective chemical potential $\mu$ associated to pion number. Consequently this

\footnotetext{
1 In fact, the pion mass $m$ and the pion decay constant $f$ appearing in this formula are modified by well-known chiral corrections, but they are not relevant for our discussion here.
} 
parameter (or equivalently the $n$ number density) can be arbitrarily chosen for any temperature $T$ by assuming that the system is surrounded by a particle bath.

Now consider the cold, $T \ll m$, classical regime of the pion gas, where the relativistic fugacity is taken small, $z=\mathrm{e}^{\beta(\mu-m)} \ll 1$. Defining as usual a thermal de Broglie wavelength,

$$
\lambda=\sqrt{\frac{2 \pi}{m T}},
$$

the number density becomes

$$
n=\frac{g z}{\lambda^{3}},
$$

and the condition of behaving in a classical way, or having low average occupation number, reads $n \ll g / \lambda^{3}$. Under this condition, (6) reduces to the formula of SackurTetrode:

$$
s=n\left(\log \frac{g}{n \lambda^{3}}+\frac{5}{2}\right),
$$

and the KSS number becomes

$$
\frac{\eta_{\chi}}{s}=\frac{240 \sqrt{2} \pi^{3}}{23} \frac{f^{4}}{m^{4}} \frac{m}{T} \frac{1}{n \lambda^{3}\left(\log \frac{g}{n \lambda^{3}}+\frac{5}{2}\right)} .
$$

It is very easy to see that in the region of validity of this formula, namely $T \ll m, n \lambda^{3} \ll g=3$ and $m \sim f$, we have $\eta_{\chi} / s \gg 1 /(4 \pi)$. Therefore, the KSS bound applies to $\chi \mathrm{PT}$ in the classical and non-relativistic limit. This model corresponds to the low energy limit of quantum chromodynamics (QCD) with $N_{f}=2$ and $N_{c}=3$. Thus this version of QCD can be considered as the UV completion of the NLSM considered here.

One of the most remarkable features of the above formula is of course the $g$ factor in the denominator. For physical pions in an isospin triplet, this degeneracy is $g=3$. It would appear that increasing the number of flavors in $\mathrm{QCD}$, which implies increasing the number of chiral Goldstone bosons (pions), eventually undermines the KSS bound according to the above formula. Nevertheless, it is well known that one cannot increase the flavor number arbitrarily in QCD without changing the derivative of the $\beta$ function, $\beta(g)=-g^{3}\left(11-2 N_{f} / 3\right) / 16 \pi^{2}$, so that the quantum field theory is presumably not well defined. In addition, the computation that we have done here is based on the NLSM based on the coset $\mathrm{SO}(g+1) / \mathrm{SO}(g)=$ $S^{g}$. However, the cosets relevant for low energy QCD are $\mathrm{SU}\left(N_{f}\right)_{\mathrm{L}} \times \mathrm{SU}\left(N_{f}\right)_{\mathrm{R}} / \mathrm{SU}\left(N_{f}\right)_{\mathrm{L}+\mathrm{R}}$. Both families of cosets meet for $g=3$ and $N_{f}=2$ but not in the general case. Thus our computation does not describe low energy QCD for $g$ different from 3 .

\section{Violating the KSS bound in the large $g$ limit}

However, the $\mathrm{SO}(g+1) / \mathrm{SO}(g)=S^{g}$ NLSM is the effective theory of the LSM based on the same groups. The LSM is a renormalizable quantum field theory (QFT) which is well defined perturbatively, although this maybe is not the case for some non-perturbative formulations because of the issue of triviality. It seems to be of help to the conjectured bound that the interaction decreases with the energy scale (the gas viscosity increases when the entropy also does), and maybe this is what will be left of the conjecture. This surely deserves further exploration.

The LSM Lagrangian is

$$
\mathcal{L}=\frac{1}{2} \partial_{\mu} \Phi \partial^{\mu} \Phi^{T}-V(\Phi)+\alpha \sqrt{g} F \sigma,
$$

where $\Phi$ is the $\mathrm{SO}(g+1)$ multiplet $\Phi=\left(\pi_{1}, \pi_{2}, \ldots, \pi_{g}, \sigma\right)$, and the potential is $V(\Phi)=-\mu^{2}|\Phi|^{2}+\lambda|\Phi|^{4}$. For appropriate $\mu^{2}$ values the potential produces spontaneous symmetry breaking from the $\mathrm{SO}(g+1)$ symmetry down to $\mathrm{SO}(g)$. As in the NLSM we have added the last term to the Lagrangian in order to break also explicitly the $\mathrm{SO}(g+1)$ symmetry, thus producing a mass $m$ for the Goldstone bosons. Defining the vacuum as $\Phi_{\mathrm{vac}}=\left(0,0, \ldots, 0, \sigma_{0}\right)$, the LSM describes the dynamics of $g$ pions of mass $m$ and a Higgs field $h=\sigma-\sigma_{0}$ with mass $m_{h}\left(m_{h}^{2}=8 \lambda g F^{2}+\right.$ $3 m^{2}$ ). The Lagrangian of the LSM in terms of these fields reads

$$
\begin{aligned}
\mathcal{L}= & \frac{1}{2} \partial_{\mu} \pi^{a} \partial^{\mu} \pi^{a}-\frac{1}{2} m^{2} \pi^{2}+\frac{1}{2} \partial_{\mu} h \partial^{\mu} h-\frac{1}{2} m_{h}^{2} h^{2} \\
& -\kappa h\left(\pi^{2}+h^{2}\right)-\lambda\left(\pi^{2}+h^{2}\right)^{2},
\end{aligned}
$$

where $\kappa=4 \lambda \sqrt{g F^{2}+m^{2} / 4 \lambda}$. The NLSM introduced in the previous section can be obtained from this one in the limit $m_{h}$ going to infinity, thus showing that the NLSM can be understood as the low energy effective theory of the LSM.

From this Lagrangian it is possible to obtain the elastic scattering amplitude for pions perturbatively. However, it is much more interesting for our purposes here to consider another kind of approach, which is the large $g$ limit. The pion elastic scattering amplitude for both the LSM and the NLSM for massive pions were found in [11]. One important thing concerning this limit is that it is defined properly only if it is taken with $g F^{2}$ fixed. Again we are interested in the non-relativistic limit. Then the relevant low energy amplitude (Weinberg theorem) is in this case $A=\left(s-m^{2}\right) /$ $g F^{2}$, and therefore we have the effective $f^{2}=g F^{2}$ fixed in the large $g$ limit. The averaged cross section in this limit is

$$
\sigma_{g}=\frac{11 m^{2}}{128 \pi\left(g^{2} F^{4}\right)}+O(1 / g),
$$

the viscosity is

$$
\eta_{g}=\frac{40 \pi^{3 / 2} g^{2} F^{4}}{11 m^{3 / 2}} \sqrt{T},
$$

and finally we have

$$
\frac{\eta_{g}}{s}=\frac{80 \sqrt{2} \pi^{3}}{11} \frac{\left(g^{2} F^{4}\right)}{m^{4}} \frac{m}{T} \frac{1}{n \lambda^{3}\left(\log \frac{g}{n \lambda^{3}}+\frac{5}{2}\right)} .
$$

This formula provides the value of the KSS parameter in the non-relativistic and classic regime of a $\mathrm{SO}(g+1)$ / 
$\mathrm{SO}(g)$ NLSM in the large $g$ limit with $g F^{2}$ fixed. As this QFT descends from the corresponding LSM, we have found in principle an explicit example of a non-relativistic system coming from a renormalizable QFT that violates the KSS bound for large enough $g$.

Is there a way out for the KSS conjecture? In principle, there could be one. As we discussed above, the NLSM incorporates processes in which two pions produce any even number of pions. Even if these processes are very much suppressed at low temperatures, $T \ll m$, they are always present. This in particular means that we cannot fix the chemical potential at will, and consequently an arbitrary value of $\mu$ will correspond in general to a metastable state outside chemical equilibrium. After some time this state will relax to the absolute stable equilibrium state $\mu=m$. Thus the density will no more be arbitrary, but it will be completely determined by the temperature alone. Interestingly enough, if $\mu=m$ then the condition $n \ll g / \lambda^{3}$ cannot be fulfilled, and our computation here is not valid anymore.

One can think of introducing a flavor chemical potential (associated to the electric or strange charge), conserved by the strong interactions. These charges of course leak through the weak interactions to electrons and muons, and are lost to the pion gas. However, for a theoretical construction of a pure sigma model system, flavor is conserved. That is, although the total number of pions varies, the relative share between the different flavors can be maintained out of chemical equilibrium with the appropriate chemical potentials, and this is enough to violate the conjectured bound.

The conclusion is that the KSS violation that we have found here applies only to states that are not chemically stable but not for a genuine thermodynamic equilibrium state, unless one can force chemical potentials associated to flavor quantum numbers upon the system. It could be the case that the same reasoning applies to the KSS bound violations found in [8] by using scaling arguments. This is because interaction terms changing the particle number probably appear in the Lagrangian density of any interacting relativistic quantum field theory satisfying the Wightman axioms.

However, there is no fundamental reason for the KSS conjecture to hold in the strong sense, for example for nonrelativistic systems of complex molecules with exponentially large degeneracy $g$, which cannot be described by the low energy limit of a relativistic QFT. In the next section we introduce a class of systems that could eventually provide a relevant physical example of KSS bound violation.

\section{Isomeric molecules provide a large degeneration factor}

There is no obvious classical gas that can be described by the non-linear sigma model, because the spontaneous symmetry breaking mechanism may not be active (Goldstone's theorem does not hold in a non-relativistic quantum field theory).

However, to overcome the KSS bound by increasing the entropy, it is sufficient to have a multicomponent gas with a large number of species. The only reason we invoked the NLSM above was that we wished to write an expression for the cross section at low energies that we could control analytically in a model that can be UV completed. If we are willing to take some uncertainty and accept an unknown cross section as a parameter instead of (9), there should be no problem in decreasing the KSS number below $1 /(4 \pi)$, provided we can arbitrarily increase the number of components in the gas.

To obtain a large number of particle species, monoatomic gases are out of the question, as the count of stable isotopes is quite limited around the stability valley. From the modest logarithmic growth of the mixing entropy with the degeneracy exposed in the denominator of (9), and the typical values for a gas in Fig. 1 and other works, which are an order of magnitude above the bound, we see that we need no less than 20000 different species to avoid the bound.

Molecular physics offers by far the largest variety of similar species in terms of stable isomers. A popular molecule family that serves our purpose for a gedanken experiment is that of the fullerenes. We sketch in Fig. 2 the well-known buckminsterfullerene $\left(\mathrm{a}_{60}\right.$ truncated icosahedron). Some two decades after their discovery, fullerenes are now copiously produced (macroscopic fractions of a gram are usual) in the form of powder, and they have also been studied in disolution. However, they are known to sublimate to a fullerene vapor at a temperature of about $750 \mathrm{~K}$. A review of the physical properties from where one may track older literature is [12]. The carbon atoms in the vertices of the truncated icosahedron $\mathrm{C}_{60}$ all fall at the surface of a sphere. Therefore, just above the sublimation temperature, a dilute fullerene gas must behave as a hard-sphere gas, with very suppressed rheologic and other properties that would blur the experimental data obtained with conventional hydrocarbon or polymer chains.

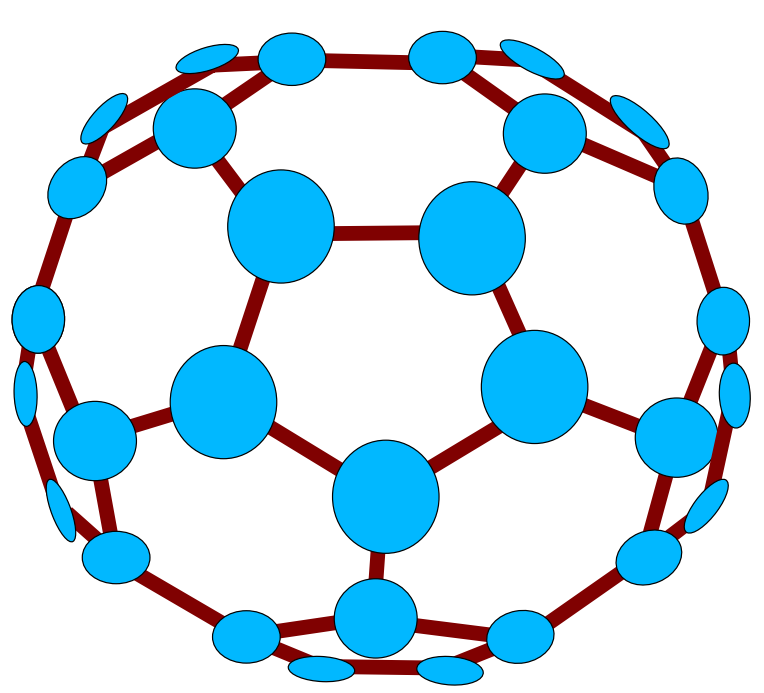

Fig. 2. Buckminsterfullerene, $\mathrm{C}_{60}$. For temperatures slightly different from the sublimation point of about $750 \mathrm{~K}$, the fullerene vapor provides a reasonable representation of a hard-sphere gas 
But how does one obtain a large number of different species? There is also a family of slightly more complicated molecules, substituted fullerenes, where one or more carbon atoms in the cage surface are replaced, such as boron-nitrogen substituted fullerenes (one can also speak of metallofullerenes, when a metal atom such as scandium or lantanum is trapped inside the cage, but these are not our focus now). Another system that could be useful is aggregated fullerenes, where one of the double carbon bonds may become simple and the extra carbon valence may be used to hook a submolecule. We refer to all these simply as substituted fullerenes, without regard of whether the counting of sites refers to a given vertex or to a given side of the fullerene.

Now, given enough substitutions, the power of combinatorial counting comes to our help. If, after accounting for the symmetries of a specific substituted fullerene, there are $N$ possible sites (or edges) for the substitution, and $M$ identical substituting atoms (perhaps not counting the first substitution), the number of different species in the gas sums up to

$$
g=\left(\begin{array}{l}
N \\
M
\end{array}\right)=\frac{N !}{M !(N-M) !},
$$

which can be made quite large (for example, with $N=12$, $M=3$, one obtains 220 isomers). In Fig. 3 we plot an example of $\mathrm{C}_{60}$ with two substitutions. The substituted fullerenes are distinguishable particles (for example by spectroscopic measurements of their vibrational lines) but have essentially equal mass and a very similar scattering cross section. Their viscosity is then essentially independent of the number of different isomers. However, the entropy grows logarithmically with the number of molecules and could eventually avoid the KSS bound. It seems, however, unlikely that the number of $\mathrm{C}_{60}$ isomers will suffice. ${ }^{2}$

But another variable comes into play: the density. The larger the molecular radius, the less dense that we can pack the molecules (and also the sooner the dilute gas approximation breaks down anyway). This makes the entropy density fall as $s \propto R^{-3}$, whereas the viscosity grows only as $\eta \propto R^{2}$. Therefore, we need an estimate of what the number of species should be for $\eta / s=1 /(4 \pi)$. This happens, assuming a certain packing coefficient $c$ defined by $n=c R^{-3}$, for cubic packing $c \sim 1 / 8$, when

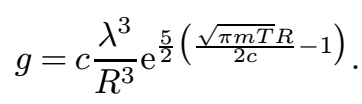

The inverse cubed radius multiplying the exponential cannot compete with the radius in the exponent, and the same applies to the $m$ and $T$ dependence. From this formula it is obvious that the number of species necessary needs to grow exponentially with their radius, mass and temperature. To minimize the required number of isomers, one therefore requires a gas that can stay cold without condensing, made of compact, light molecules. Helium satisfies all three conditions, but fails to violate the KSS bound by a factor 8.8 ; therefore, we would need some $\left.2400^{\frac{s}{n}}\right|_{\text {critical }}$ isomers!

\footnotetext{
${ }^{2}$ We thank D. Son for a rough estimate indicating that this particular gas will still fail to violate the bound.
}

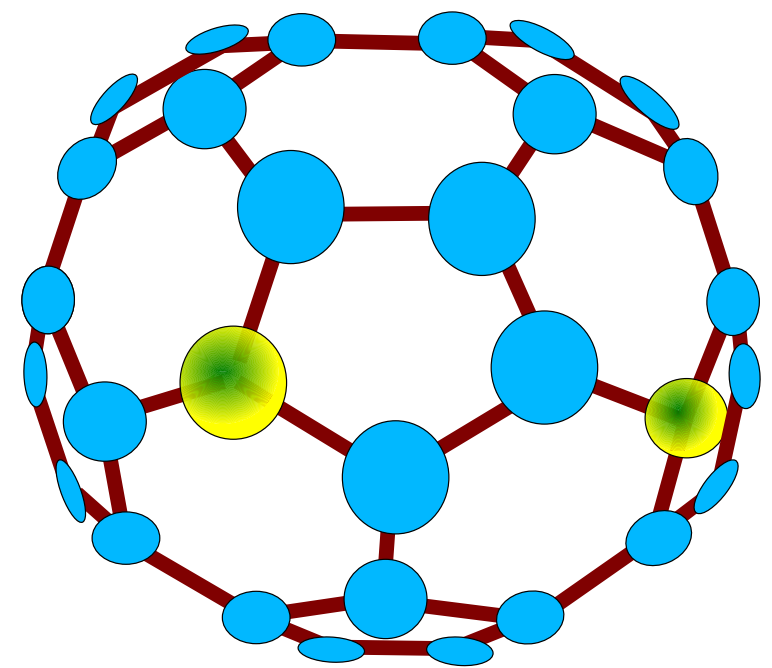

Fig. 3. Example of doubly-substituted $\mathrm{C}_{60}$

We can further reduce (16) by assuming that the mass of a molecule scales with the radius as a power, $m \propto R$, $R^{2}, R^{3}$ for families of molecules such as polymers, fullerenes and globular proteins, respectively. Furthermore, the boiling point is also known to scale as a power (typically smaller than 1) of the mass number; see Fig. 4 for an example.

Expressing everything in terms of the number of atoms in the molecule, we find a typical value of

$$
g \propto N^{-7 / 2} \mathrm{e}^{c N^{7 / 6}}
$$

since the number of possible substitutions will typically grow as (in Stirling's approximation)

$$
g \propto N ! \simeq \mathrm{e}^{N \log N}
$$

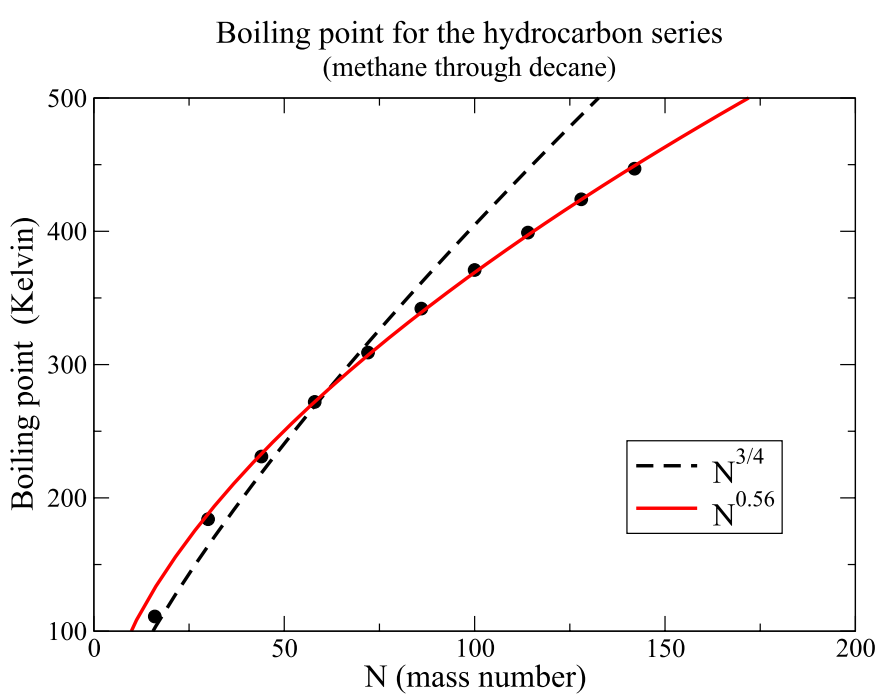

Fig. 4. Boiling point for the hydrocarbon series. It can be fit by a function of the form $T_{\mathrm{b}}(K)=28 N^{0.56}$, growing with the molecule size because of the increased polarizability 
we see that most families of substituted molecules will fail, since they will start further from the bound than helium, but increasing their size provides barely, if at all, enough gain in isomer number. The candidate molecule family needs to satisfy the four conditions of compactness, coldness, lightness, and large isomer number. Helium satisfies the first three optimally, but it fails to violate the bound by a factor about 8.8 at its critical point, and being chemically inert, it fails the fourth condition.

\section{Discussion and outlook}

On physical grounds, it has been argued before [13] that the applicability of the Navier-Stokes equations restricts the possible range of viscosities, and, moreover, the viscosity should be larger than the entropy density up to a constant factor, on the grounds of the uncertainty principle alone. The novelty [2] is now that for a whole class of field theories, those with a gravity dual, the factor is precisely $1 /(4 \pi)$. No example is known with a smaller value.

From this note, it is apparent that a physically realizable non-relativistic system can indeed evade this KSS bound. We have shown this explicitly and as a matter of principle with the non-linear sigma model, and we have given an example of a multicomponent gas with hundreds to thousands of isomers, composed of substituted fullerenes, and given the conditions that can provide a physical violation of the bound. In the end one can argue that this, or any other molecular gas made of stable isotopes, may be completed by QED and QCD with the matter content $e^{-}$, $u, d$ (and perhaps $s$ ). Since there are multiple such fluids associated to the many scales and eigenvalues of the combined Hamiltonian, the question arises as to for which of them, or whether at all, a precisely stated KSS conjecture would apply.

In spite of the results found here concerning the $\eta / s$ ratio at the non-relativistic classic regime of the $\mathrm{SO}(g+1) /$ $\mathrm{SO}(g)$ NLSM in the large $g$ limit, it is possible that the KSS bound could be maintained in the weak sense, because of the particle production present in any consistent
QFT. However, multicomponent gases of complex carbon molecules as the ones presented here could violate the bound in the strong sense.

It is finally interesting to speculate on a possible measurement of $\eta / s$ for the hadron gas in relativistic heavyion collisions. The reason is that, if the traditional ideas of Hagedorn, implying exponential growth of the hadron species when approaching the phase transition, also being built into the concept of Regge trajectories, would be correct, then $\eta / s$ would dramatically fall near the phase transition. A finite measurement of $\eta / s$ at RHIC, FAIR or the LHC, when combined with (16) or a suitable generalization thereof, could be used as an upper bound on the number of hadron resonances. To our knowledge this has never been attempted.

Acknowledgements. We thank T. Cohen and D.T. Son for enthusiastic correspondence about possible improvements to the original preprint. This work has been supported by grants FPA 2004-02602, 2005-02327, PR27/05-13955-BSCH (Spain).

\section{References}

1. P. Kovtun, D.T. Son, A.O. Starinets, JHEP 0310, 064 (2003) [hep-th/0309213]

2. P. Kovtun, D.T. Son, A.O. Starinets, Phys. Rev. Lett. 94, 111601 (2005) [hep-th/0405231]

3. S. Gavin, M. Abdel-Aziz, Phys. Rev. Lett. 97, 162302 (2006) [nucl-th/0606061]

4. T. Schafer, cond-mat/0701251

5. A. Dobado, F.J. Llanes-Estrada, hep-ph/0609255, to appear in Eur. Phys. J. C

6. J.W. Chen, E. Nakano, Phys. Lett. B 647, 371 (2007)

7. S. Laha, T. Gangopadhyay, M. Bagchi, M. Dey, J. Dey, M. Sinha, S. Ray, hep-ph/0702086

8. T.D. Cohen, hep-th/0702136

9. S. Weinberg, Physica A 96, 327 (1979)

10. J. Gasser, H. Leutwyler, Ann. Phys. 158, 142 (1984)

11. A. Dobado, J. Morales, Phys. Rev. D 52, 2878 (1995)

12. O. Gunnarson, Rev. Mod. Phys. 69, 575 (1997)

13. P. Danielewicz, M. Gyulassy, Phys. Rev. D 31, 53 (1985) 